\title{
Long-term safety and efficacy of adalimumab in psoriasis: a multicentric study focused on infections (connecting study)
}

\author{
Alessandra Narcisi ${ }^{1,2}$, Nicoletta Bernardini ${ }^{3}$, Diego Orsini ${ }^{4}$, Magda D'Agostino $^{5}$, Catia De Felice ${ }^{6}$, \\ Alessandro Di Stefani ${ }^{5}$, Valentina Carboni ${ }^{7}$, Antonio Costanzo ${ }^{1,2}$, Claudio Mastroianni $^{8}$ \\ ${ }^{1}$ Humanitas Clinical and Research Center, Skin Pathology Lab, Rozzano-Milan, Italy \\ 2Dermatology Unit, Department of Biomedical Sciences, Humanitas University, Rozzano-Milan, Italy \\ ${ }^{3}$ Dermatology Unit "Daniele Innocenzi", University of Rome "Sapienza", I Faculty of Medicine and Surgery, Polo Pontino, \\ Terracina (LT), Italy \\ ${ }^{4}$ IFO San Gallicano Institute (IRCCS), Infectious Dermatology and Allergology Unit, Rome, Italy \\ ${ }^{5}$ Fondazione Policlinico Universitario Agostino Gemelli IRCCS, Rome, Italy \\ ${ }^{6}$ IFO San Gallicano Institute (IRCCS), Psoriasis Unit, Rome, Italy \\ 'Dermatology Unit ASL Rm-H, Frascati (RM), Italy \\ ${ }^{8}$ Infectious Disease Unit, Department of Public Health and Infectious Diseases, University of Rome "Sapienza", Policlinico Umberto \\ I Hospital of Rome, Italy \\ Adv Dermatol Allergol 2020; XXXVII (3): 428-434 \\ DOI: https://doi.org/10.5114/ada.2020.96910
}

\begin{abstract}
Introduction: This Italian multicenter retrospective study evaluated safety and efficacy of the anti-TNF drug, adalimumab, in a cohort of patients affected by tuberculosis (TB), hepatitis B virus (HBV), hepatitis C virus (HCV) or human immunodeficiency virus (HIV). Psoriasis is an autoimmune disease affecting around $3 \%$ of the Italian population and associated with several comorbidities, including arthritis, cardio-metabolic diseases and depression. In its moderate-to-severe form, psoriasis profoundly impairs quality of life of patients.

Aim: Therefore, these patients deserve systemic treatments including conventional DMARDS (disease modifying anti-rheumatic drugs) and biologics. Management of moderate and severe psoriasis patients affected by relevant infections such as TB, HBV, HCV and HIV may be difficult because of the toxicity of the conventional systemic treatment.

Material and methods: The CONNECTING study analysed 28 moderate to severe psoriasis patients infected by TB, HBV, HCV and HIV who were treated with adalimumab for up to 96 weeks together with respective prophylactic treatment.

Results: We observed a rapid decrease in PASI (psoriasis area severity index) reaching a $75 \%$ improvement in $91 \%$ of patients. Some of these patients $(n=9)$ were also affected by arthritic comorbidity. The patients experienced a rapid decrease in pain, measured by pain VAS (visual analogic scale) that reached 0 in all of them. Monitoring of the respective infection did not show any worsening or reactivation of infection or any severe adverse events during the entire observation period.

Conclusions: Adalimumab is effective and safe in patients affected by these important infections.
\end{abstract}

Key words: psoriasis, infection, biological drugs, adalimumab, efficacy, safety.

\section{Introduction}

Psoriasis ( $\mathrm{PsO}$ ) is an auto-immune disease affecting $1-3 \%$ of the world population [1]. Psoriasis is a chronic relapsing condition that requires continuous care for its physical and psychological implications. The severity of this disease depends, in fact, not only on its extent on the skin (measured by PASI, psoriasis area and severity index), but also by its impact on the patient's quality of life (measured by DLQI, dermatological life quality in-

Address for correspondence: Diego Orsini MD, IFO San Gallicano Institute (IRCCS), Infectious Dermatology and Allergology Unit, Via Elio Chianesi 52, 00123 Rome, Italy, mobile phone: +39 335/1774185, e-mail: diegorsini@gmail.com Received: 20.04.2018, accepted: 5.09.2018. 
dex). The impact of cutaneous plaques on physical and mental functions of patients is similar to that of other severe chronic diseases (such as cancer), with high levels of stress, poor self-esteem, increased rates of mood disorders and detrimental effects on social functioning, interpersonal relationships and productivity [2, 3]. In addition, plaque psoriasis is only a part of the psoriatic disease, which often implies a diffuse inflammation of other organs and structures, and is associated with several comorbidities (metabolic syndrome, non-alcoholic fatty liver disease, depression, arthritis) [4].

Treatment strategies for severe or refractory/recalcitrant PsO recommended by International and National Guidelines are based on disease-modifying drugs (DMARDs), such as methotrexate and cyclosporine, small molecules such as apremilast (a PDE4 inhibitor) and dimethyl fumarate or biologic drugs such as tumour necrosis factor (TNF) inhibitors (adalimumab, infliximab, etanercept, golimumab) or anti-interleukin therapies (ustekinumab, secukinumab, ixekizumab) $[5,6]$. To date, adalimumab is the most frequently used biological agent in PsO and psoriatic arthritis (PsA). Since February 2016, adalimumab and the other biological therapies have been approved by the European Medicines Agency for the treatment of moderate to severe chronic plaque psoriasis as first-line treatment in adult patients who are candidates for systemic therapy and not only after previous inadequate response/intolerance to systemic DMARDs [7].

TNF- $\alpha$, a pleiotropic cytokine which regulates several inflammatory reactions and immune functions through the control of cellular processes, plays a central role in the pathogenesis of PsO. The over-expression of TNF is able to promote pro-inflammatory conditions and is involved in several pathogenetic mechanisms such as the accumulation of leukocytes in the inflamed skin, induction of dermal vascular changes and itching, and inflammation and joint-bone damage [8]. TNF inhibitors represent effective therapeutic options for the treatment of PsO, significantly improving signs and symptoms and reducing the disease activity. These drugs represent an outstanding innovation in the treatment of inflammatory immuno-mediated diseases and an important option for modifying the natural course and the unfavourable progression of these diseases [9-11].

The efficacy and safety of these molecules is well demonstrated in clinical studies, but also in real life. In fact, there are a lot of studies that demonstrate a minor development of infections in patients affected by PsO, PSA and rheumatoid arthritis (RA), treated with antiTNF- $\alpha$ in relation to DMARDS. There are also case studies that confirmed their efficacy and safety in patients affected by non-active hepatitis B virus (HBV), hepatitis $\mathrm{C}$ virus (HCV), human immunodeficiency virus (HIV) and tuberculosis (TB).

\section{Aim}

The aim of this retrospective study was to collect data on efficacy and safety of PsO patients affected by HBV, HCV, HIV and/or TB infections, treated with adalimumab in real life. In our opinion, real life data reflect the daily clinical practice, different from randomized clinical trials, where stricter inclusion/exclusion criteria are adopted for selecting study populations.

\section{Material and methods}

\section{Patients and study design}

CONNECTING was a retrospective non-interventional investigation study performed by analysing clinical databases of psoriasis patients affected by TB, or HBV, or $\mathrm{HCV}$ or HIV, treated with adalimumab in 6 centres of the Lazio region. Anti-TNF agent (adalimumab) was administered to patients according to the label (following EU guidelines) [7]. Inclusion criteria included: adult male or female patients diagnosed with moderate to severe psoriasis or psoriatic arthritis currently being treated with adalimumab for at least 12 months (with the exception of two HIV patients who reached week 12 and 24, respectively); documented seropositivity for TB, HBV, HCV or HIV. Diagnosis of psoriasis and psoriatic arthritis was clinical. Moderate to severe psoriasis was diagnosed according to the criterion of the 'rule of tens' by Finlay (BSA involved $>10 \%$ or PASI score $>10$ or DLQI score $>10$ ) and all PsA patients met the CASPAR (Classification criteria for Psoriatic Arthritis) criteria for the classification of PSA $[12,13]$. The data recording was designed in order to have demographic and anamnestic data, concomitant diseases, time of evolution, current and previous biologic treatments, Psoriasis Area Severity Index (PASI) score during the observational period. We also recorded baseline data, including the serological status of bacteria or virus, serum liver tests, chest $\mathrm{X}$-ray.

\section{Outcome measures}

To evaluate the efficacy of the anti-TNF agent (adalimumab), the response rate of a 75\%, 90\% and 100\% improvement in the PASI score (respectively PASI 75, PASI 90 and PASI 100) was calculated at each time point. Safety was assessed by recording the occurrence of serious adverse events and infection reactivation/worsening.

\section{Results}

\section{Baseline demographic and clinical characteristics}

A total of 26 patients were included in the CONNECTING study. Patient clinical and demographic characteristics are presented in Table 1. There was a higher prevalence of male patients. Thirteen patients were active smokers, 6 ex-smokers and 7 never smoked; arterial hypertension was present in 13 patients, diabetes in 5, dyslipid- 
Table 1. Incidence of infections in our patients

\begin{tabular}{lcc}
\hline Parameter & Freq. & Percent \\
\hline TB & 11 & 37.93 \\
\hline HBV & 9 & 31.03 \\
\hline HCV & 6 & 20.69 \\
\hline HIV & 3 & 10.34 \\
\hline Total & 29 & 100 \\
\hline
\end{tabular}

emia in 15, metabolic syndrome in 6 and depression in 5 . Twenty-two patients were naïve to biologics and 4 were subjected to previous biologic treatment (2 etanercept, 1 infliximab and 1 ustekinumab). Nine patients had also a diagnosis of psoriatic arthritis (by CASPAR criteria).

\section{TBC infection}

Eleven patients ( 9 males and 2 females, mean age 55.18 years) were positive to TB - Quantiferon assay (IGRA), all of them were symptomless for active TB infection, 10 of them were negative at chest $X$-ray and one with doubtful chest $X$-ray but negative chest CT scan. One patient was co-infected by HIV and another co-infected by HBV. The mean PASI in these patients was $22.69 \pm 8.07$ at baseline. Patients were subjected to prophylactic treatment with Isoniazid at $300 \mathrm{mg} /$ day for 1 month before starting adalimumab and for additional 5 months in cotreatment. Adalimumab treatment was administered for at least 1 year ( 6 patients reached 2 years of continuous treatment). At week 48, the mean PASI in these patients was $0.89 \pm 1.28$ (Table 2). None of these patients showed TB reactivation.

\section{Hepatitis B virus infection}

Nine patients ( 5 males and 4 females, mean age 59 years) were positive to HBV infection, all of them were positive for anti-HBc Abs, 2 were HBsAg positive, 5 antiHBs positive, 2 HBV-DNA positive and 3 anti-HBe positive (Table 3). Mean AST value was $32.1 \pm 16.76$, mean ALT 34.5 \pm 19 .3. One patient was co-infected by TB. The mean PASI in these patients was $18.44 \pm 4.14$ at baseline. Patients with HBV-DNA and HBsAg positivity $(n=2)$ were subjected to tenofovir treatment, while 2 patients Anti-HBe and Anti-HBs positive were co-treated with Lamivudine $100 \mathrm{mg} /$ die. Adalimumab treatment was administered for at least 1 year ( 5 patients reached 2 years of continuous treatment). At week 48, the mean PASI in these patients was $1.09 \pm 1.44$ with 7 patients reaching PASI 100. None of these patients showed HBV worsening during the course of adalimumab treatment.

\section{Hepatitis C virus infection}

Six patients ( 3 males and 3 females, mean age 58 years) were positive to HCV infection, all of them were

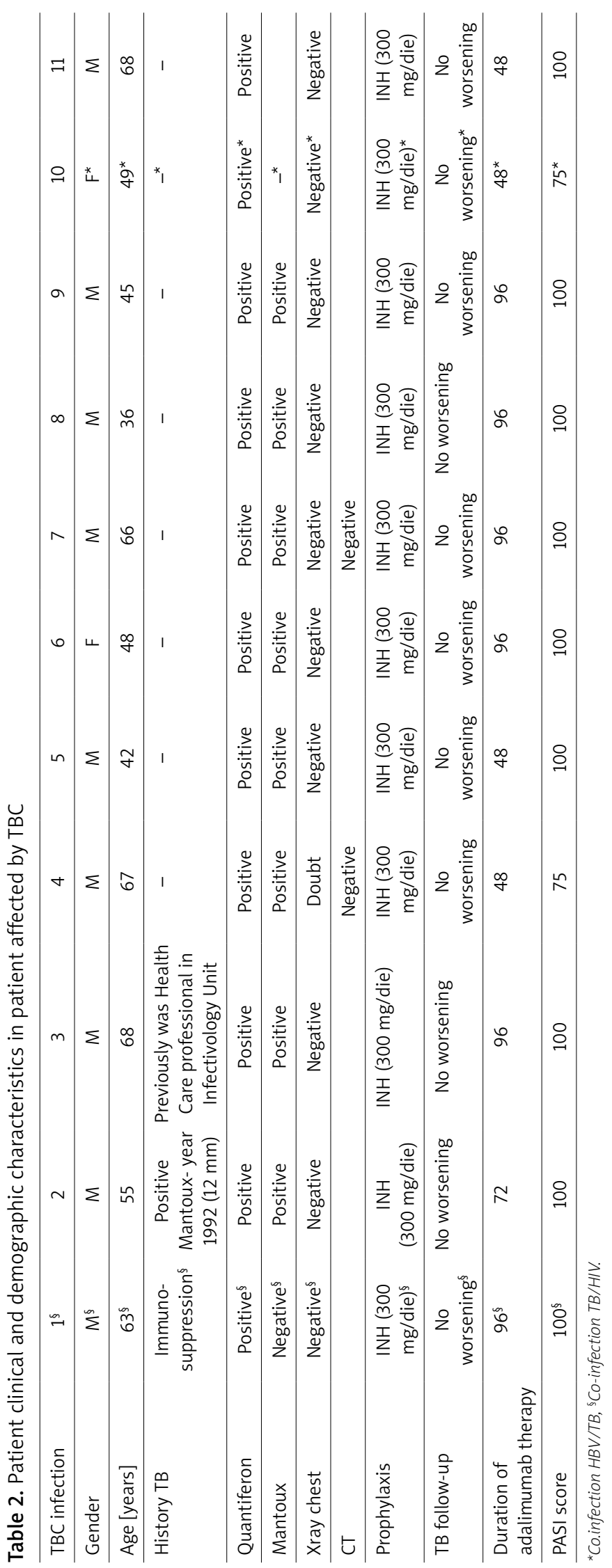


Table 3. Patient clinical and demographic characteristics in patient affected by HBV

\begin{tabular}{|c|c|c|c|c|c|c|c|c|c|}
\hline HBV infection & 1 & 2 & 3 & 4 & 5 & 6 & 7 & 8 & $9^{*}$ \\
\hline Gender & M & M & $\mathrm{F}$ & M & M & $\mathrm{F}$ & M & $\mathrm{F}$ & $\mathrm{F}$ \\
\hline Age [years] & 45 & 59 & 62 & 60 & 72 & 51 & 72 & 61 & 49 \\
\hline $\mathrm{HBsAg}$ & Positive & Negative & Negative & Positive & Negative & Negative & Negative & Negative & Negative \\
\hline HBcAb (IgG) & Positive & Positive & Positive & Positive & Positive & Positive & Positive & Positive & Positive \\
\hline $\mathrm{HBsAb}$ & Positive & Positive & Positive & Positive & Negative & Negative & Negative & Negative & Positive \\
\hline $\begin{array}{l}\text { HBV-DNA } \\
\text { [copies/ml] }\end{array}$ & Positive & Negative & Negative & Positive & Negative & Negative & Negative & Negative & Negative \\
\hline $\mathrm{HBeAg}$ & Positive & Negative & Negative & Negative & - & Negative & - & - & - \\
\hline HbeAb (IgG) & Positive & Positive & Positive & Negative & - & Negative & - & - & - \\
\hline GOT & 50 & 15 & 12 & 45 & 46 & 50 & 37 & 11 & 23 \\
\hline GPT & 45 & 18 & 15 & 52 & 51 & 62 & 40 & 10 & 18 \\
\hline$\gamma$-GT & 43 & 20 & 22 & 55 & 48 & 36 & 51 & 13 & 15 \\
\hline Total bilirubin & - & - & - & - & 1.2 & 1.5 & 1.1 & 0.33 & 0.45 \\
\hline $\begin{array}{l}\text { Alkaline } \\
\text { phosphatase }\end{array}$ & - & - & - & - & - & 48 & 300 & 257 & 115 \\
\hline LDH & & & & & & & & & 150 \\
\hline $\begin{array}{l}\text { Antiviral } \\
\text { prophylaxis } \\
\text { /therapy for } \\
\text { HBV }\end{array}$ & Tenofovir & $\begin{array}{l}\text { Lamivudine } \\
\text { (100 mg/die) }\end{array}$ & $\begin{array}{l}\text { Lamivudine } \\
\text { (100 mg/die) }\end{array}$ & Tenofovir & - & - & - & - & - \\
\hline $\begin{array}{l}\text { HBV follow- } \\
\text { up }\end{array}$ & $\begin{array}{c}\text { No } \\
\text { worsening }\end{array}$ & $\begin{array}{c}\text { No } \\
\text { worsening }\end{array}$ & $\begin{array}{c}\text { No } \\
\text { worsening }\end{array}$ & $\begin{array}{c}\text { No } \\
\text { worsening }\end{array}$ & $\begin{array}{c}\text { No } \\
\text { worsening }\end{array}$ & $\begin{array}{c}\text { No } \\
\text { worsening }\end{array}$ & $\begin{array}{c}\text { No } \\
\text { worsening }\end{array}$ & $\begin{array}{c}\text { No } \\
\text { worsening }\end{array}$ & $\begin{array}{c}\text { No } \\
\text { worsening }\end{array}$ \\
\hline $\begin{array}{l}\text { Duration of } \\
\text { adalimumab } \\
\text { therapy } \\
\text { [weeks] }\end{array}$ & 96 & 96 & 96 & 72 & 72 & 96 & 48 & 96 & 48 \\
\hline $\begin{array}{l}\text { PASI } \\
\text { response }\end{array}$ & PASI 100 & PASI 100 & PASI 75 & PASI 100 & PASI 100 & PASI 100 & PASI 100 & PASI 100 & PASI 75 \\
\hline
\end{tabular}

${ }^{*}$ Co.infection HBV/TB.

positive for anti-HCV Abs, 1 patient was also positive to HCV-RNA and showed second grade fibrosis at fibroscan (Table 4). Mean AST value was $63.8 \pm 17.6$, mean ALT 57.5 \pm 16 . One patient was co-infected with HIV. The mean PASI in these patients was $17.5 \pm 6.75$ at baseline. No prophylactic treatment was given to these patients. Adalimumab treatment was administered for at least 1 year (5 patients reached 2 years of continuous treatment). At week 48 , the mean PASI in these patients was $0 \pm 0$ with 7 patients reaching PASI 100. None of these patients showed HBV worsening during the course of adalimumab treatment.

\section{HIV infection}

Three patients (3 males, mean age: 52.3 years) were positive to HIV infection, all of them were positive for anti-HIV Abs, in none of them HIV-RNA was detectable, mean CD4+ cell count was $648 \pm 220$ cells/ml (Table 5 ).
One patient was co-infected with TB. The mean PASI in these patients was $21.67 \pm 6.51$ at baseline. All these patients were co-treated with HAART therapy starting before adalimumab treatment. As for adalimumab efficacy, 1 patient is at week 12 with a $60 \%$ improvement in his PASI, another patient reached PASI 75 at week 24, a third patient reached PASI 100 at week 96. None of these patients showed HIV worsening during the course of adalimumab treatment.

\section{Overall PASI clinical response rate in TB, HBV, HCV and HIV patients}

The clinical response was evaluated both as mean PASI and as the percentage of patients obtaining PASI 75, 90 or 100 over the 2-year follow-up period. Figure 1 A shows the reduction in PASI score in these patients. The baseline PASI value was 19.98 that progressively decreased to 1.59 at week 24, 0.74 at week $48,0.46$ at week 72 and 0.63 
Table 4. Patient clinical and demographic characteristics in patient affected by HCV

\begin{tabular}{|c|c|c|c|c|c|c|}
\hline HCV infection & 1 & 2 & 3 & 4 & 5 & $6^{\circ}$ \\
\hline Gender & M & $\mathrm{F}$ & M & $\mathrm{F}$ & $\mathrm{F}$ & $M^{\circ}$ \\
\hline Age [years] & 53 & 61 & 58 & 66 & 53 & $57^{\circ}$ \\
\hline $\mathrm{HCV} A \mathrm{~b}$ & Positive & Positive & Positive & Positive & Positive & Positive $^{\circ}$ \\
\hline HCV RNA & Positive & Negative & Negative & Negative & Negative & Negative $^{\circ}$ \\
\hline GOT & 85 & 55 & 43 & 86 & 55 & $59^{\circ}$ \\
\hline GPT & 72 & 52 & 51 & 82 & 48 & $40^{\circ}$ \\
\hline$\gamma$-GT- & 78 & 72 & 68 & 71 & 36 & $77^{\circ}$ \\
\hline Total bilirubin & & & & & 2 & $1.1^{\circ}$ \\
\hline Alkaline phosphatase & - & - & - & - & 309 & $-{ }^{\circ}$ \\
\hline Fibroscan & $\begin{array}{l}\text { Second grade } \\
\text { Fibrosis (F2) }\end{array}$ & - & - & - & - & $-^{\circ}$ \\
\hline HCV follow-up & No worsening & No worsening & No worsening & No worsening & No worsening & No worsening ${ }^{\circ}$ \\
\hline $\begin{array}{l}\text { Duration of adalimumab } \\
\text { therapy [weeks] }\end{array}$ & 72 & 72 & 72 & 72 & 96 & $24^{\circ}$ \\
\hline PASI response & PASI 100 & PASI 100 & PASI 100 & PASI 100 & PASI 100 & PASI $75^{\circ}$ \\
\hline
\end{tabular}

${ }^{\circ} \mathrm{Co}$-infection HIV/HCV.

Table 5. Patient clinical and demographic characteristics in patient affected by HIV

\begin{tabular}{|c|c|c|c|}
\hline HIV infection & $1^{\S}$ & $2^{\circ}$ & 3 \\
\hline Gender & $M^{\S}$ & $M^{\circ}$ & M \\
\hline Age [years] & $63^{\S}$ & $57^{\circ}$ & 37 \\
\hline HIV Ab & Positive ${ }^{\S}$ & Positive $^{\circ}$ & Positive \\
\hline HIV RNA & Negative ${ }^{\S}$ & Not detectable ${ }^{\circ}$ & Not detectable \\
\hline CD4+ [cells/ $\mu l]$ & $400^{\S}$ & CD4+: 725 (31\%) cell/ $/ 1^{\circ}$ & CD4+: 820 (36\%) cell/ $/ \mu \mathrm{l}$ \\
\hline HAART therapy & $Y_{e s}^{\S}$ & $\mathrm{Yes}^{\circ}$ & Yes \\
\hline HIV follow-up & No worsening $\$$ & No worsening ${ }^{\circ}$ & No worsening \\
\hline Duration of adalimumab therapy [weeks] & $96^{\S}$ & $24^{\circ}$ & 12 \\
\hline PASI response & PASI $100^{\S}$ & PASI $75^{\circ}$ & $60 \%$ PASI improvement at 12 week \\
\hline
\end{tabular}

${ }^{8}$ Co-infection TB/HIV, ${ }^{\circ} \mathrm{Co}$-infection HIV/HCV.

at week 96. PASI 75, 90 and 100 was reached by $91.67 \%$, $83.33 \%$ and $83.33 \%$ of patients, respectively (Figure 1 B). The speed of PASI response in these patients was similar to that observed in clinical trials and in real-life experience. At week $16,64 \%, 36 \%$ and $28 \%$ of patients reached PASI 75, 90 and 100, respectively (Figure 1 B), which confirmed the efficacy of adalimumab in this special set of patients. In patients also showing articular disease (9 patients), the pain VAS was analysed. Similarly to that observed for PASI, we observed a rapid decrease in mean pain VAS from 4.07 at baseline to 0.94 at week 16, 0.67 at week 24, 0.15 at week 48, 0.08 at week 72 and 0 at week 96 (Figure 2). Analysis of PASI response to adalimumab in the different subgroups did not show any significant differences suggesting that these infections do not affect adalimumab efficacy in psoriasis (Figure 3).

\section{Safety}

The overall safety profile of adalimumab in this subset of high-risk patients was excellent. During the entire study period we did not observe any serious adverse events. In addition we did not observe any reactivation or worsening of the pre-existing infection, which further confirmed the safety profile of adalimumab.

\section{Discussion}

Psoriasis is a complex, genetically determined syndrome whose skin phenotype represents only the "tip of the iceberg". It is often associated with arthropathy and numerous comorbidities. Based on the latest research, the notion of non-random association of these pathologies is being more evident, and the researchers are trying to iden- 

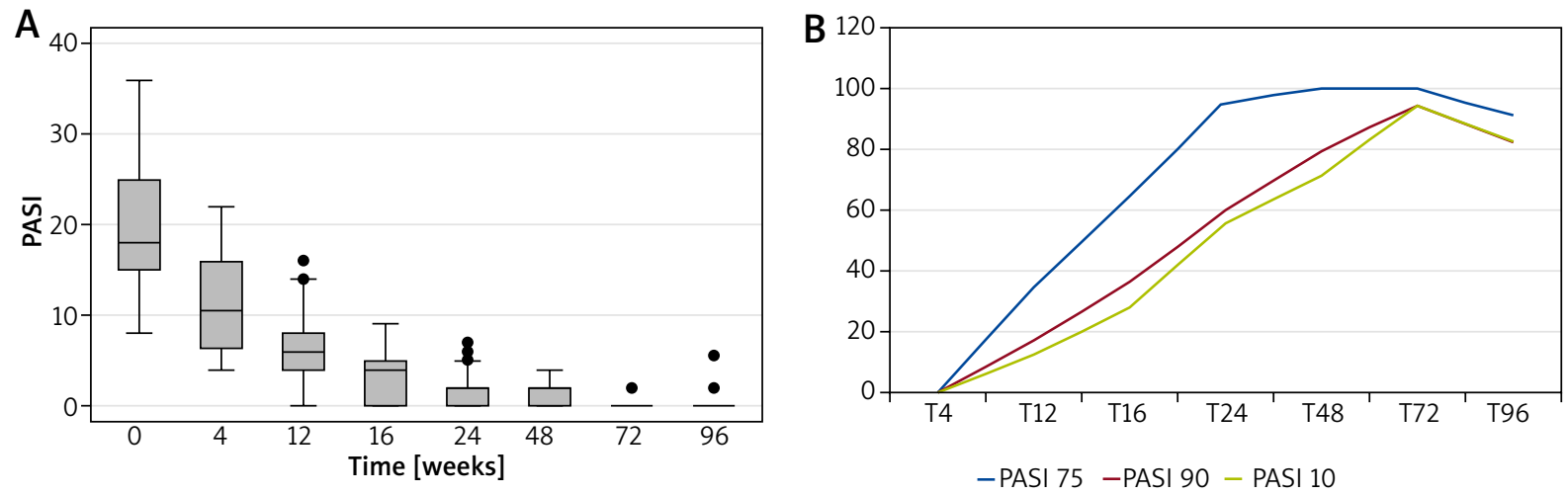

Figure 1. A - Median PASI per week, B - PASI 75, 90 and 10 per week

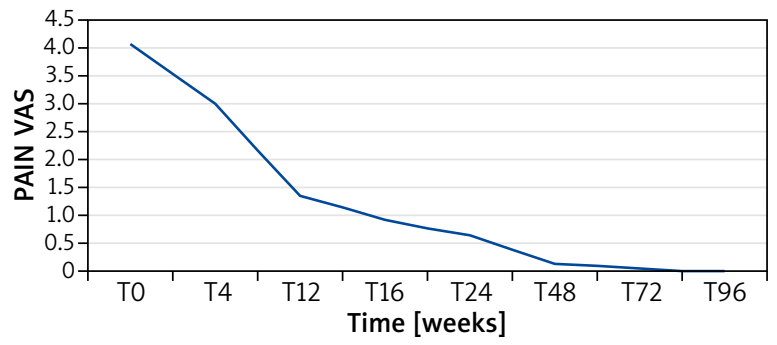

Figure 2. PAIN VAS per week

tify pathogenetic links and common immune alterations that determine the inflammation of different organs. It is therefore essential to the psoriasis patient as "systemic" at the outset of the disease and treat it effectively and early to avoid the progression of systemic and joint damage. Conventional systemic therapies, used in patients with moderate to severe disease for long periods, may adversely affect comorbidities. Hence, the need to use new molecules, which act on target cytokines or pro-inflammatory molecules that play a key role in the pathogenesis of the disease: biological drugs. These molecules often provide a good result in terms of effectiveness, but clinicians have yet some doubts as to safety of these molecules, especially in selected populations such as in the elderly, or in adults infected by hepatitis, tuberculosis or HIV. The data currently available in the literature on these subjects largely derive from European or non-European case reports or registers, and are difficult to compare with each other for a number of reasons: the absence of long-term studies, the heterodynamics of the populations analyzed (age, geographic area, sex, comorbidity), heterogeneity of treated diseases (psoriatic arthritis, rheumatoid arthritis, ankylosing spondylitis), heterogeneity of the therapies used (different biological drugs and associations with other drugs), arbitrary criteria used to define the severity of the associated infection [14].

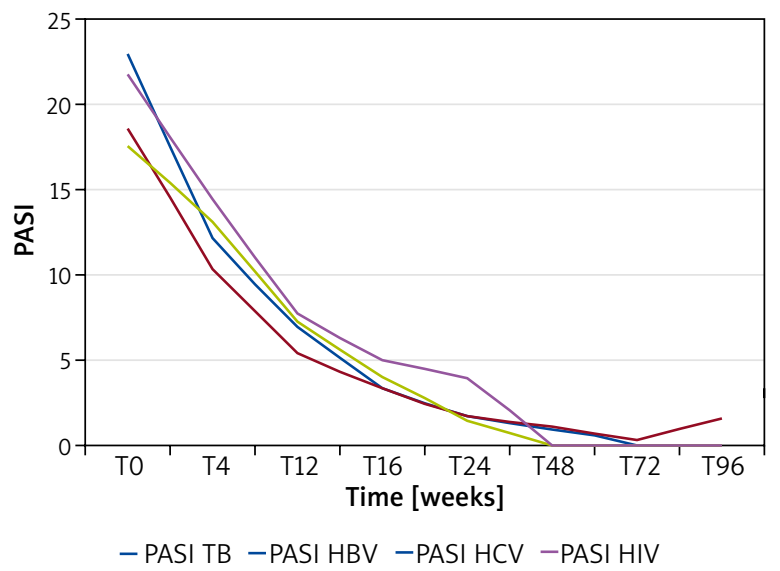

Figure 3. PASI per week in single infection

The main outcome measures examined in this retrospective study, carried out in a routine clinical setting using the data of a clinical database of $\mathrm{PsO}$ patients infected by hepatitis, tuberculosis and/or HIV, treated with adalimumab in 6 Italian centres, were the drug efficacy and safety. This is the largest Italian study on efficacy and safety of adalimumab in this selected population.

The results of the retrospective study conducted on 28 patients for a period of 96 weeks, correspond with the data of international literature.

Considering the efficacy and safety outcomes based on PASI response and the monitoring of reactivation or exacerbation of infections (HBV, HCV, HIV or TB), in our experience, adalimumab is a good effective and safe treatment for this selected group of patients. The efficacy results are similar to or even higher than those reported in clinical trials, with a rapid decrease in PASI that remained stable in the entire observational period. The overall safety profile of adalimumab in this subset of high-risk patients was excellent. During the entire study period we did not observe any serious adverse events. In 
addition we did not observe any reactivation or worsening of the pre-existing infection, which further confirmed the safety profile of adalimumab.

\section{Conclusions}

These results confirm that adalimumab is a valid therapy for the long-term management of severe-tomoderate $\mathrm{PsO}$ in patients with chronic infections. This study confirms that real-life data represent an important source of information on long-term treatment, efficacy and safety of adalimumab in selected groups of patients, usually excluded from randomized controlled trials.

Obviously, the risk-benefit ratio must guide medical practice, and so we could affirm that the use of biological drugs in patients infected by HBV, HCV, HIV or TB, is reserved to that cases in which the benefit for the patient outweighs the risk of infection's reactivation, and we recommend a strict monitoring of these patients by a dermatologist in collaboration with infectiologists and other specialists if necessary (such as a pneumologist or gastroenterologist). It is also necessary to make it clear and to create shared guidelines on screening, monitoring and treatment of associated infections during adalimumab therapy.

\section{Conflict of interest}

The authors declare no conflict of interest.

\section{References}

1. Lande R, Botti E, Jandus C, et al. The antimicrobial peptide LL37 is a T-cell autoantigen in psoriasis. Nat Commun 2014; 5: 5621. Erratum in: Nat Commun 2015; 6: 6595.

2. Rapp SR, Feldman SR, Exum ML, et al. Psoriasis causes as much disability as other major medical diseases. J Am Acad Dermatol 1999; 41: 401-7.

3. Eghlileb AM, Davies EEG, Finlay AY. Psoriasis has a major secondary impact on the lives of family members and partners. Br J Dermatol 2007; 156: 1245-50.

4. Lubrano E, Cantini F, Costanzo A, et al. Measuring psoriatic disease in clinical practice. An expert opinion position paper. Autoimmun Rev 2015; 14: 864-74.

5. Nast A, Gisondi P, Ormerod AD, et al. European S3-Guidelines on the systemic treatment of psoriasis vulgaris--Update 2015--Short version--EDF in cooperation with EADV and IPC. J Eur Acad Dermatol Venereol 2015; 29: 2277-94.

6. Blandizzi C, Gionchetti P, Armuzzi A, et al. The role of tumour necrosis factor in the pathogenesis of immune-mediated diseases. Int I Immunopathol Pharmacol 2014; 27 (1 Suppl): 1-10.

7. Furst DE, Keystone EC, Braun J, et al. Updated consensus statement on biological agents for the treatment of rheumatic diseases. Ann Rheum Dis 2012: 71 (Suppl. 2): i2-45.

8. Blandizzi C; Societa' Italiana Di Farmacologia. Position paper. Equivalenza terapeutica di classe dei farmaci inibitori del tumour necrosis factor: analogie e differenze farmacologiche.
9. Raval K, Lofland JH, Waters H, et al. Disease and treatment burden of psoriasis: examining the impact of biologics. J Drugs Dermatol 2011; 10: 189-96.

10. Finlay AY. Current severepsoriasis and the rule of tens. Br J Dermatol 2005; 152: 861-7.

11. Taylor W, Gladman D, Helliwell P, et al.; the CASPAR Study Group. Classification criteria for psoriatic arthritis: development of new criteria from a large international study. Arthritis Rheum 2006; 54: 2665-73.

12. Bongartz T, Sutton AJ, Sweeting MJ, et al. Anti-TNF antibody therapy in rheumatoid arthritis and the risk of serious infections and malignancies: systematic review and meta-analysis of rare harmful effects in randomized controlled trials. JAMA 2006; 295: 2275-85.

13. Dixon WG, Watson K, Lunt M, et al. British Society for Rheumatology Biologics Register Control Center Consortium; British Society for Rheumatology Biologic Register. Serious infection following anti-TNF alpha-therapy in patients with rheumatois arthritis: lessons from interpreting data from observational studies. Arthritis Rheum 2007; 56: 2896-904.

14. Curtis JR, Patkar N, Xie A, et al. Risk of serious bacterial infections among rheumatoid arthritis patients exposd to tumor necrosis factor alpha antagonists. Arthritis Rheum 2007; 56 : 1125-33. 